\title{
An Integrative Business Case Utilizing An Online Database
}

Louise Miller, Robert Morris University, USA

\begin{abstract}
This paper describes a unique business case that is an integration of topics in accounting information systems, managerial accounting, economics, and marketing into a single comprehensive student project on the internet. The project described gives students experience using an online database and php coding to interact with this database. The problem also involves demand functions and profit maximization with elementary calculus. The information system used is a website that assists a company employee in setting prices of products for optimal profit, given information concerning demand function parameters, costs, and marketing options. Although this project was used in an accounting information systems course, it could also be utilized in other courses such as introductory database management systems, managerial accounting, or introductory information technology. The project can be easily modified to align with the experience and sophistication of the students.
\end{abstract}

Keywords: Accounting Information Systems; Internet; Optimization; Databases

\section{INTRODUCTION}

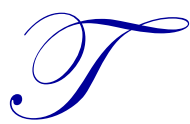

echnology in all forms, especially internet technology, is becoming integral and essential to accounting systems for both large and small businesses. The AICPA has defined one of the core competencies of students as being "Technologically Adept" or "able to utilize and leverage technology in ways that add value to clients, customers and employers" (AICPA, 1996). The word "technology" in an accounting setting has traditionally referred to students being adept with spreadsheets, accounting software packages such as QuickBooks, or database software such as Microsoft Access. Databases have become extremely important in business because they are the foundation of almost all management and accounting information systems used by companies, particularly large Enterprise Resource Planning (ERP) systems. Simkin (2011) points out that teaching database skills effectively is an integral part of the typical university accounting curriculum as well as a professional mandate. Pillsbury and Wang (2011) believe that for students to really understand the importance and use of relational databases, they need to experience the application of database management concepts, not just receive abstract coverage of the topic in a textbook and lecture. However, as Badua (2008) concludes, the scope of accounting information systems is greater and more profound than the technical operations of accounting software or database programs, such as Microsoft Access. Also, we are now being rapidly immersed into internet technology at all levels and in all areas of accounting from financial reporting and XBRL to e-commerce systems, online ERP systems and other outsourced accounting-related services. The internet allows small businesses to remotely manage and store information and access customized software, services, or information from virtually any device with internet access. As the internet evolves, it is transforming accounting. The move to the internet has the potential to increase collaboration and efficiency and reduce costs for businesses, but it also presents challenges to AIS educators.

So the accounting profession is now immersed in the internet age, and while many required accounting information systems courses and textbooks introduce database design and accounting software development (Romney \& Steinbart, 2003; Kay \& Ovlia, 2012), projects involving the actual integration of database projects with accounting concepts in a hands-on student project on the internet are uncommon. Macur (2011) analyzed course content from 56 accounting information systems courses and found that only 17 had database projects, and of those, 15 used Microsoft Access. This illustrates that Microsoft Access is the database software of choice in AIS courses 
and that the internet does not come into play when assigning accounting database projects to students. For example, Stewart (2011) describes a typical database management project that was being used in the classroom utilizing Access 2000.

Unlike most AIS courses, this paper describes a student project assignment that involves a database that is integrated with a website. This project gives students actual "real world" experience and knowledge of an internetbased accounting system. Although this project was used in an accounting course, it could also be utilized in other courses such as introductory database management systems, web design, or introductory information technology. The project can be easily modified to align with the experience and sophistication of the students taking the course. The project described here was used in an introductory accounting information system class taken primarily by sophomore and junior accounting and finance majors, so no prior knowledge of database systems or systems development was assumed or expected. The project also does not assume any prior knowledge of website design, query languages, or software coding. Adapting the project to upper-level courses for students with a greater knowledge of internet programming would be straightforward. This project can also be used as a class demonstration in marketing, microeconomics or managerial accounting courses to illustrate real-world application of the topics of cost analysis for pricing decisions and profit maximization.

This integrative case described here illustrates profit analysis for a small manufacturing operation and requires the students to use elementary calculus to find the equation for the optimal selling price of a product. Students are given information concerning costs, product demand functions, and marketing options. This case gives students insight into the calculus of optimization used in business problems and could also be used in a microeconomics course to illustrate the concepts of economic equilibrium for price and quantity in a competitive market. This case can also be used in marketing courses to illustrate that effective pricing strategies and analysis of various options that affect demand can greatly affect profits and ultimately affect competitive advantage.

The fundamental idea of this project is to create a simple application for a widget company in a website that uses a database that can be accessed via the internet. The instructor of the course initially sets up a web hosting account and registers an internet domain name. This does not require as large of an investment as it would have in the past because virtually all web hosting companies provide all the services and tools required for a sophisticated internet site that is integrated with a database in their basic level web hosting service. For the project described here, Yahoo Web hosting was used at a cost of only $\$ 14.95$ per month. This low cost includes unlimited space, multiple users with selective access levels, and multiple databases. The fundamental process for the instructor in this project is setting up the web hosting environment and then giving all students taking the course access as administrators to be allowed to create or modify an accounting information system website. Administrator rights give students complete access to the database tools and admin areas to give them knowledge and experience with the management of an actual accounting software implementation on the internet. (Although the instructions and descriptions here are specific to Yahoo Web hosting, all web hosting services provide similar services and administrative access web pages.)

Most enterprise databases used in accounting information systems are built with sophisticated and powerful database software such as MS SQL Server or Oracle DBMS. These databases are uploaded to the web and software, such as SQL - a query and data manipulation language - is used to access and modify the database. PHP is an HTML-embedded scripting language designed for web development to produce dynamic web pages. PHP code is embedded into the HTML source document and interpreted by a web server with a PHP processor module, which generates the web page document. PHP can be deployed on web servers on almost every operating system and platform free of charge. SQL statements to interact with the database are embedded into the PHP code, and that code displays the results on a web page. PHP is installed on more than twenty million websites and one million web servers. Due to its popularity, virtually all web hosting services provide free tools for PHP coding and database interaction. The phpMyAdmin tool is a free open-source utility that is made available through the Yahoo Web hosting Control Panel. This tool handles the administration of MySQL over the Web. It can create and import databases, create and alter database tables and fields, and execute any SQL statement. 


\section{PROJECT ENVIRONMENT DESCRIPTION}

The fictitious LM Widget Company is a chain of stores located across the United States. The student's job is to create (or modify) a simple website that determines the optimal pricing for a product. Information about the product is contained in a database that resides within the web hosting account that is set up for the course. All students have administrator access to the web hosting account where they each create their own website and code for implementing their unique assignment. After implementing their website, each student uses the website to calculate the optimal price of a product.

The fundamental steps for project set-up by the instructor of the course prior to assigning the project to the students are:

1. Register a domain name for the course's website. (e.g. www.LMwidgetcompanyAISclass.com)

2. Sign up for the basic web hosting service with Yahoo Web hosting for this domain.

3. Using Yahoo's password manager, create a username and password for student access to the account. Grant administrator rights for this username so that all students in the class can access the web hosting control panels and functions.

4. In Yahoo Web Hosting, install the phpMyAdmin tool and use it to import spreadsheets containing the data for the initial LM Widget Company database. This installation is achieved with just a few steps and will require the assignment of a separate username and password to access the phpMyAdmin website.

5. Go to the filemanager and create a sample directory to store the web pages (code) for the LM Widget Company website.

In a pre-assignment classroom demonstration, the instructor enters the Yahoo Web hosting administrator panel for the course website. (Any modern university classroom provides internet access.) The instructor demonstrates how to $\log$ in to the website control area and also how to enter the phpMyAdmin website, which allows viewing of the actual databases that reside in the website. The phpMyAdmin website also has many other functions available related to the databases, such as inserting or deleting records, assigning primary keys, sorting, or changing the actual structure of the database design. This classroom demonstration can reinforce many topics that have been covered in prior business courses such as database design.

Following the classroom demonstration, the students are assigned the project, which can be submitted over several weeks. The assignment is described in the following section.

\section{STUDENT PROJECT ASSIGNMENT DESCRIPTION}

First, students are given instructions for accessing the web hosting site. They are told to go to yahoo.com and log in with the student username and password given to them by the instructor. The file manager is where files and folders are manipulated, stored, and organized. One folder contains the database code files (the code to run the database functions) and the students are to create their own student folder (subdirectory) in the main folder of the file manager. The simple website for the LM Widget Company consists of two web pages - a product pricing page with an input form and a product pricing results page - and the students are instructed to copy these files into their own subdirectory:

1. ProductPricingFormHTML.htm

2. ProductPriceCalcResults.php

The students will now have their own LM Widget Company website. (For advanced courses, in which students are familiar with HTML, the main page creation can be a requirement, or advanced enhancements can be required here.) In the web hosting account, a mysql database is set up called LMWidgetCompany. This is the database that the code in the LM Widget Company website will interact with when employees use the website. The LMWidgetCompany database has a product list table. To look at the database and the table in it, the students can use the phpMyAdmin utility, which they access with their student username and password. Figure 1 illustrates the display of the ProductList table in this utility. 


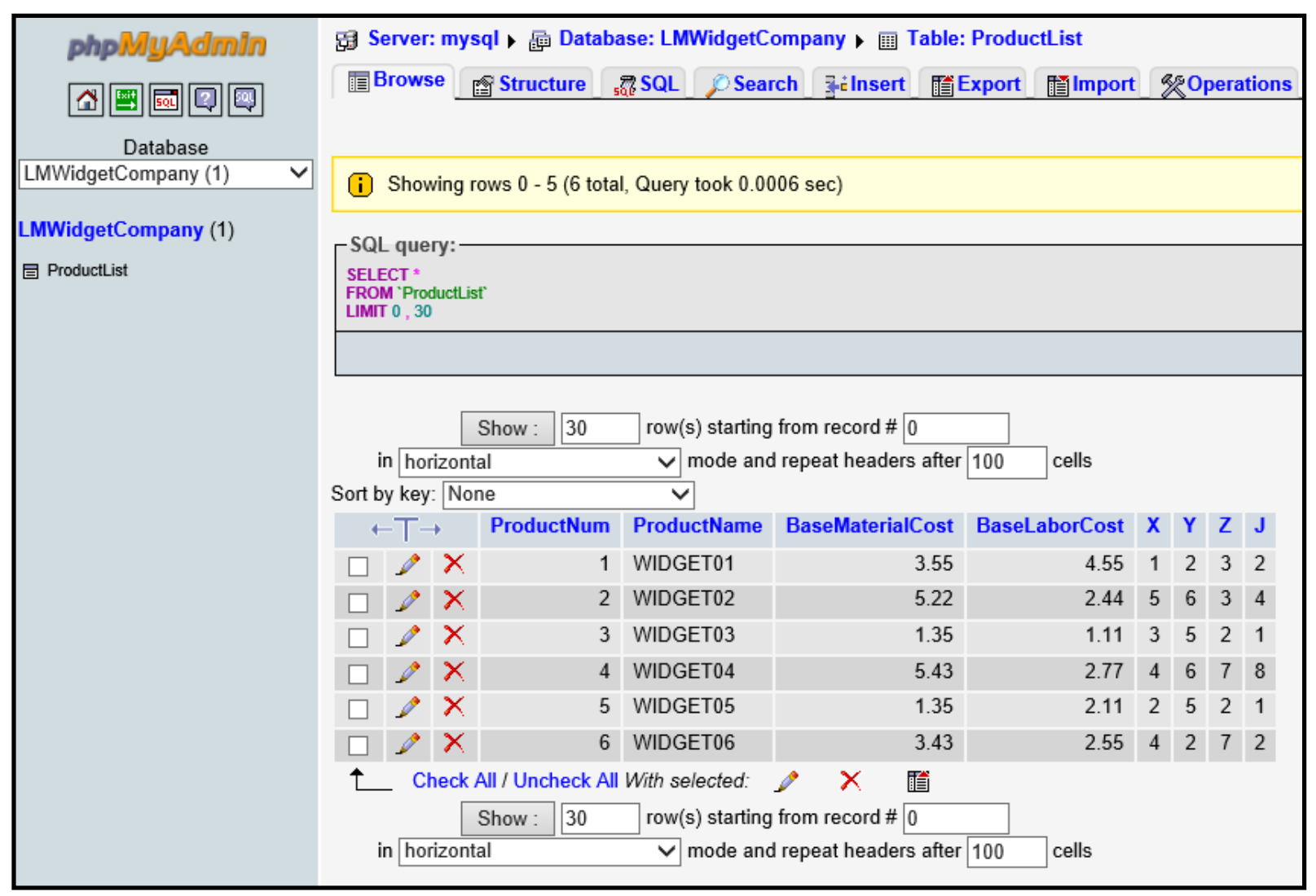

Figure 1: Display of the Product List Table in the PhpMyAdmin Utility

In this project, the managers of LM Widget Company want a website that will quickly calculate optimal prices for products via a website. This website will access the centralized database created by the marketing department and the user will enter other parameters for the product design on a web page. The website will use the database information and the inputs from the web page to calculate the optimal price for a product to maximize profit for the company.

The database contains values for the following in the database for each product they manufacture: Product name, Base Variable Material Cost per unit, Base Variable Labor Cost per unit, and Demand parameters X, Y, Z, and $\mathrm{J}$.

The marketing department has determined that the demand function for a product is given by:

$\mathrm{Q}=\mathrm{A}-\mathrm{BP}$

where $\mathrm{Q}$ is quantity demanded and $\mathrm{P}$ is Price.

The value $\mathrm{A}$ is a function of the advertising done for the product and is given by:

$\mathrm{A}=\mathrm{L} * \mathrm{X}+\mathrm{Y}$

where $\mathrm{L}$ is the level of Advertising done given by High $=3$, Medium $=2$ and Low $=1$.

This indicates that the value of A will be higher for more advertising done and therefore the quantity demanded will be higher. The value of B in the demand function is one of location:

$\mathrm{B}=\mathrm{W} * \mathrm{Z}+\mathrm{J}$ 
where $\mathrm{W}$ indicates location, which is set to various values according to area of the country where the product is to be sold, causing the quantity demanded to vary with region.

Given the expressions for $\mathrm{A}$ and $\mathrm{B}$, the demand function $\mathrm{Q}=\mathrm{A}-\mathrm{BP}$ becomes:

$\mathrm{Q}=(\mathrm{L} * \mathrm{X}+\mathrm{Y})-(\mathrm{W} * \mathrm{Z}+\mathrm{J}) * \mathrm{P}$

In other words, the quantity demanded is a function of the demand parameters $\mathrm{X}, \mathrm{Y}, \mathrm{Z}$ and $\mathrm{J}$ and the price.

The website will also allow the employee to specify the level of customization and time frame of delivery for the product, which affect the cost of labor and materials.

Total Variable Cost per unit V = Total Variable Material Cost per unit + Total Variable Labor Cost per unit

Total Variable Material Cost per unit $=\mathrm{C} * \mathrm{M}_{\mathrm{BASE}}$

where $\mathrm{C}$ is customization level and $\mathrm{M}_{\mathrm{BASE}}$ is base material cost per unit.

Total Variable Labor Cost per unit $=(\mathrm{C}+\mathrm{T}) * \mathrm{~L}_{\mathrm{BASE}}$

where $\mathrm{T}$ is the timeframe and $\mathrm{L}_{\mathrm{BASE}}$ is the base labor cost per unit.

Substituting these expressions into the equation for Total Variable Cost per unit:

Total Variable Cost per unit $=\mathrm{V}=\mathrm{C} * \mathrm{M}_{\mathrm{BASE}}+(\mathrm{C}+\mathrm{T}) * \mathrm{~L}_{\mathrm{BASE}}$

Students are given the above information and asked to find an expression for the optimal price for maximum profit and the equation for the maximum profit.

\section{Solution to Profit Maximization} written as:

The optimal price is now determined with elementary calculus. The profit equation for a product can be

Profit $=$ Sales - Total Variable Costs $-\mathrm{F}$

where $\mathrm{F}$ is fixed expenses. becomes:

Substituting price times quantity, $\mathrm{P} * \mathrm{Q}$ for sales and $\mathrm{V}^{*} \mathrm{Q}$ for the total variable costs, the profit equation

Profit $=\mathrm{P}^{*} \mathrm{Q}-\mathrm{V} * \mathrm{Q}-\mathrm{F}=(\mathrm{P}-\mathrm{V}) * \mathrm{Q}-\mathrm{F}$

Since $\mathrm{Q}=\mathrm{A}-\mathrm{BP}$, this becomes:

Profit $=(\mathrm{P}-\mathrm{V})^{*}(\mathrm{~A}-\mathrm{BP})-\mathrm{F}$

Or

Profit $=-\mathrm{BP}^{2}+\mathrm{PA}-\mathrm{VA}+\mathrm{VBP}-\mathrm{F}$

To find the optimal price for maximum profit, the derivative of the profit is taken and set equal to zero:

$\mathrm{dProfit} / \mathrm{dP}=-2 \mathrm{BP}_{\text {optimal }}+\mathrm{A}+\mathrm{VB}=0$ 
$\mathrm{P}_{\text {optimal }}=(\mathrm{A}+\mathrm{VB}) / 2 \mathrm{~B}$

Now the previous expressions for $\mathrm{A}, \mathrm{B}$, and $\mathrm{V}$ can be substituted, resulting in the final expression for optimal price:

$\mathrm{P}_{\text {optimal }}=\left((\mathrm{L} * \mathrm{X}+\mathrm{Y})+\left(\mathrm{C} * \mathrm{M}_{\mathrm{BASE}}+(\mathrm{C}+\mathrm{T}) * \mathrm{~L}_{\mathrm{BASE}}\right)(\mathrm{W} * \mathrm{Z}+\mathrm{J})\right) /(2(\mathrm{~W} * \mathrm{Z}+\mathrm{J}))$

To summarize, the optimal price $\mathrm{P}_{\text {optimal }}$ for a product is a function of the parameters stored in the database (base labor cost $\mathrm{L}_{\mathrm{BASE}}$, base material cost $\mathrm{M}_{\mathrm{BASE}}$, and demand parameters $\mathrm{X}, \mathrm{Y}, \mathrm{Z}$ and $\mathrm{J}$ ) and the selections made by the employee on the website (location $\mathrm{W}$, advertising level L, customization level C, and time frame T).

Substituting this optimal price expression into the profit equation, the maximum profit is given by:

Profit $_{\text {MAXIMUM }}=-\mathrm{B}_{\text {optimal }^{2}}{ }^{2} \mathrm{P}_{\text {optimal }} \mathrm{A}-\mathrm{VA}+\mathrm{VB}_{\text {optimal }}-\mathrm{F}$

where $\mathrm{A}=\mathrm{L} * \mathrm{X}+\mathrm{Y}, \mathrm{B}=\mathrm{W} * \mathrm{Z}+\mathrm{J}$, and $\mathrm{V}=\mathrm{C} * \mathrm{M}_{\mathrm{BASE}}+(\mathrm{C}+\mathrm{T}) * \mathrm{~L}_{\mathrm{BASE}}$

Or:

Profit $_{\text {MAXIMUM }}=-(\mathrm{W} * \mathrm{Z}+\mathrm{J})\left(\left(\left((\mathrm{L} * \mathrm{X}+\mathrm{Y})+\left(\mathrm{C} * \mathrm{M}_{\mathrm{BASE}}+(\mathrm{C}+\mathrm{T}) * \mathrm{~L}_{\mathrm{BASE}}\right)(\mathrm{W} * \mathrm{Z}+\mathrm{J})\right) /(2(\mathrm{~W} * \mathrm{Z}+\mathrm{J}))\right)^{2}+(((\mathrm{L} * \mathrm{X}+\mathrm{Y})+\right.$ $\left.\left.\left(\mathrm{C} * \mathrm{M}_{\mathrm{BASE}}+(\mathrm{C}+\mathrm{T}) * \mathrm{~L}_{\mathrm{BASE}}\right)(\mathrm{W} * \mathrm{Z}+\mathrm{J})\right) /(2(\mathrm{~W} * \mathrm{Z}+\mathrm{J}))\right)\left((\mathrm{L} * \mathrm{X}+\mathrm{Y})+\left(\mathrm{C} * \mathrm{M}_{\mathrm{BASE}}+(\mathrm{C}+\mathrm{T}) * \mathrm{~L}_{\mathrm{BASE}}\right)(\mathrm{W} * \mathrm{Z}+\mathrm{J})\right)-$ $\left(\mathrm{C} * \mathrm{M}_{\mathrm{BASE}}+(\mathrm{C}+\mathrm{T}) * \mathrm{~L}_{\mathrm{BASE}}\right)(\mathrm{L} * \mathrm{X}+\mathrm{Y})-\mathrm{F}$

Students are asked to implement their functions for optimal price and maximum profit in the php code for the Optimal Product Price Results web page. Profit at the optimal price is displayed as well as the profit obtained at a "price guess" by the user. Both of these profit values use the fixed expenses value entered by the user. The "price guess" and resulting profit using that guess are useful to the student to observe that guesses either below or above the optimal price results in a lower profit than the maximum possible.

The complete website is shown in Figures 2 and 3. The main web page shown in Figure 2 is a form where the employee enters the options for product. After entering information for the product pricing request, the "Calculate Optimal Price and Profit" button is pressed and the product pricing results page is displayed, as shown in Figure 3. The code modules ProductPricingFormHTML.htm and ProductPriceCalcResults.php, shown in Figures 4 and 5, handle form display and the product price and profit calculations that are done on the web pages. 
$\sim$ Welcome to the LM Widget Company!

Find the Optimal Price for a Product $\sim \sim$

Enter Product Name:

WIDGET01

Choose Location:

South Central $\checkmark$

Choose Customization Level:

Level $1 \mathrm{~V}$

Choose Advertising Level:

Level $2 \mathrm{~V}$

Choose Timeframe:

Rush V

Enter Total Fixed Expenses:

250.00

Enter your guess for the Product Price:

11.00

Calculate Optimal Price and Profit

Figure 2: Main Web Page for the LM Widget Company

\section{Here are your results:}

For Product: WIDGET01

Optimal Price $=\$ 8.74$

Profit at the Optimal Price $=\$ 751.33$

Your Price Guess $=\$ 11.00$

Profit at your Price Guess $=\$ 680.00$

Your guess for the price was too high!

Figure 3: Optimal Product Pricing Results Web Page 
$<$ title>Widget Price Calculation</title>

$<$ body bgcolor="\#ccccff" link="\#3333FF" vlink="\#666633" text="\#000000"><br $>\langle$ br $><b>$

$<$ span style="font-size:20"> Welcome to the LM Widget Company! $<$ br $></$ span $><b r>$

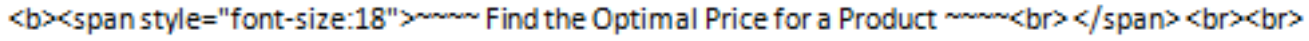

$<$ form action="http://louiseannette.com/FILESforLMWidgetCompanyPaper/ProductPriceCalcResults.php"

method="post">

Enter Product Name $<\mathrm{br} />$

<input name="searchproductName" type=" "text" size $=" 30 " /\rangle\langle\mathrm{br} /\rangle\langle\mathrm{br} /\rangle$

Choose Location $<\mathrm{br} />$

$<$ select name="Location">

<option value $=" 1 ">$ West $</$ option>

$<$ option value $=" 2$ " $>$ Midwest $</$ option $>$

<option value $=" 3$ ">South $</$ option>

$<$ <ption value $=" 4$ ">South Centra|</option>

$<$ option value $=" 5$ " $>$ Northeast $</$ option $>$

$</$ select $><\mathrm{br} /><\mathrm{br} />$

Choose Customization Level $<\mathrm{br} />$

<select name="CustomizationLevel">

<option value="1">Level $1</$ option>

<option value $=" 2$ " $>$ Level $2<$ /option>

<option value=" 3 "> Level $3</$ option>

$</$ select $><b r /><b r />$

Choose Advertising Level $<\mathrm{br} />$

<select name="AdvertisingLevel">

<option value $=" 1 ">$ Level $1<$ /option>

$<$ coption value $=" 2 ">$ Level $2</$ option>

$<$ option value $=" 3$ "> Level $3<$ /option>

$</$ select $><\mathrm{br} /><\mathrm{br} />$

Choose Timeframe $<\mathrm{br} />$

<select name="Timeframe" >

<option value $=" 1 ">$ Normal</option $>$

<option value $=" 2 ">$ Rush $</$ option>

<option value=" 3 ">Extreme Rush</option>

$</$ select $><\mathrm{br} /><\mathrm{br} />$

Enter Total Fixed Expenses $<\mathrm{br} />$

<input name="FixedExpenses" type="float" />

$\langle\mathrm{br} /\rangle\langle\mathrm{br} /\rangle\langle\mathrm{br}\rangle$

Enter your guess for the Product Price: $<\mathrm{br} />$

<input name="priceguess" type="float" />

$<\mathrm{br} /><\mathrm{br} />$

<input type="submit" name="submit" value="Calculate Optimal Price and Profit"/>

$</$ form $>$

Figure 4: HTML Code for the Product Pricing Request Form 
$<$ html $><$ head $><$ title>Results $</$ title $></$ head $>$

<body><body bgcolor="\#99cccc" link="\#3333FF" vlink="\#666633" text="\#000000">

$<$ b $><$ span style="font-size: 24 " $>$ Here are your results: $<b r\rangle</$ span $><b r><b r>$

$<$ ?

// create short variable names for inputs from the FORM

\$searchproductName = \$_POST['searchproductName'];

\$Timeframe $=$ \$_POST['Timeframe'];

\$AdvertisingLevel = \$_POST['AdvertisingLevel'];

\$Customization Level = \$_POST['Customization Level'];

\$Location = \$_POST['Location'];

\$priceguess = \$_POST['priceguess'];

\$FixedExpenses = \$_POST['Fixed Expenses'];

//CONNECT TO MYSQL

\$mysql_access = mysql_connect('mysql', 'pillow_lady', 'abcabc12');

if (!'\$mysql_access) \{die ('Could not connect: '. mysql_error(1));

//CONNECT TO DATABASE

$\$ \mathrm{db}=$ "LMWidgetCompany";

mysql_select_db(\$db, \$mysql_access) or die('Could not connect to database...' . mysql_error());

//echo 'Connected successfully to database ....... <br><br>';

Stablename='ProductList'; // Name of the table

\$results=array();

\$sql= 'SELECT * FROM ProductList WHERE (ProductName = "'.\$searchproductName."' )';

Sresult = mysql_query(\$sql) or die('Error: '. mysql_error());

while(\$_a_row = mysql_fetch_array $($ Sresult $))$ array_push(\$ results, Sa_row);

if(count(Sresults))

$\{/ /$ IF THERE ARE RESULTS DISPLAYTHEM

foreach(\$results as $\$ r) \quad\{$

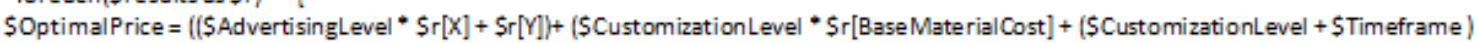

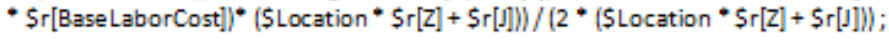

echo(' For Product: '.\$searchproductName. '<br><br');

\$formattedNum = number_format (\$OptimalPrice, 2);

echo(' Optimal Price = \$'.\$formatted Num.'<br><br');

$\$ A=\$ A d v e r t i s i n g L e v e l * \$ r[X]+\$ r[Y]$;

$\$ B=\$$ Location * $\$ r[Z]+\$ r[]] ;$

\$V = \$Customization Level * \$r[Base MaterialCost] + (\$Customization Level + \$Timeframe $) * \$ r[B a s e ~ L a b o r$ Cost]

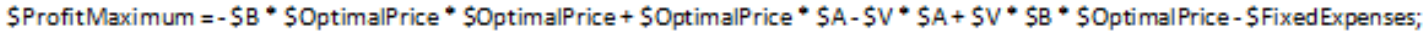

SformattedNum = number_format (\$ProfitMaximum, 2);

echo('Profit at the Optimal Price $=\$$ '. SformattedNum. '<br $><b r><b r>$ ');

SformattedNum = number_format(\$priceguess, 2);

echo(' Your Price Guess = \$'.\$formattedNum. '<br><br>');

\$ProfitatGuess = -\$B* \$priceguess * \$priceguess + \$priceguess * \$A - \$V*\$A+ \$V*\$B* \$priceguess-\$FixedExpenses;

\$formattedNum = number_format (\$ProfitatGuess, 2$)$;

echo(' Profit at your Price Guess= \$'.\$formattedNum. '<br $><b r>1)$;

if (\$ProfitatGuess < \$ProfitMaximum)

\{ if (\$priceguess < \$OptimalPrice)

\{ echo('Your guess for the price was too low!'); \}

else \{echo('Your guess for the price was too high!'); \}

\}else \{echo('Your guess for the price was correct!'); \}

\} \} else fecho('Somy-That product was not found in the database'); \}

?)

$</$ body $>$

$</ \mathrm{html}>$

Figure 5: Code for Optimal Price and Profit Calculations and Display of the Results Web Page 
The code in ProductPriceCalcResults.php implements the pricing and profit functions and the students can be given a file without the functions and asked to modify the code with their solution to the optimization problem. The code is pulling the information from the database table and using that to calculate the optimal price. More advanced students in information technology courses can be asked to create the product pricing results page code themselves or implement additional functionality rather than modifying existing code. This part of the project facilitates understanding of the basics of query languages and fundamental SQL statements, as well as their relationship to databases.

Finally, students are instructed to summarize the capabilities of the LM Widget Company's information system, explaining in detail the interactions and functions of the database located on the internet. They are also asked to explain what capabilities would need to be added to make this a complete pricing system, such as forms to enter new records into the database and functions for generating pricing reports for management.

\section{CONCLUSION}

This paper describes a comprehensive student project on the internet used in an accounting information systems course. The project described is a pricing system using an online database which could be easily modified to align with the experience and sophistication of the students. The project is a combination of class demonstration and a student assignment that integrates the topics of internet databases, query languages, and the calculus of profit maximization. This project could also be used in introductory or upper level managerial accounting courses because it highlights the topic of cost analysis for pricing decisions. Optimization problem solutions are critically important for the analysis of decisions related to resource allocations and the analysis of future profit projections. This case is also appropriate for use in marketing or introductory microeconomics courses. Most importantly, this case illustrates to students that in real-world complex business operations, internet technology is important and that decisions regarding uncertainties and the allocation of resources are significant and affect the profit outcomes of a business.

The specific learning objectives (functional competencies) of this project are for students to:

1. Gain an understanding of the fundamentals of database design and implementation on the internet

2. Understand the basics of query languages and fundamental SQL statements, as well as their relationship to databases and web hosting services

3. Understand that profit optimization analysis can be a valuable decision-making process for business managers concerned with future business success

4. Understand that in real-world manufacturing operations, decisions regarding the allocation of resources affect the profit outcomes of a company

5. Gain an understanding of the fundamentals of the calculus of optimization used in business problems

6. Understand that in a competitive market, there is an economic equilibrium for price and quantity demanded and that effective pricing strategies and analysis of various options that affect demand and costs can greatly affect profits

By mapping the project activities to functional competencies and directly measuring multiple outcomes in these activities, these competencies can be assessed in the course as prescribed in the accounting department's assessment plan.

\section{AUTHOR INFORMATION}

Dr. Louise Miller has a PhD in Management Science, an MBA and a BS degree in Electrical Engineering. Her research interests include accounting information systems and the integration of technology into accounting education. Dr. Miller also enjoys painting and web site design and programming. She teaches for Robert Morris University in Moon Township, Pennsylvania. E-mail: millerl@rmu.edu 


\section{REFERENCES}

1. American Institute of Certified Public Accountants (AICPA). 1996. Information technology competencies in the accounting profession: AICPA implementation strategies for IFAC international education guideline no. 11: Implications for education and practice. New York, NY: AICPA.

2. Badua, F. (2008). Pedagogy and the PC: Trends in the AIS curriculum. Journal of Education for Business, 83(5), 259-264.

3. Kay, D., \& Ovlia, A. (2012). Accounting information systems. New York, NY: Prentice Hall.

4. Macur, K. M. (2011). The curricular content of accounting information systems. Review of Business Information Systems (RBIS), 2(3), 11-20.

5. Pillsbury, C. M., \& Wang, T. J. (2011). Supplemental materials for database management system knowledge and skills in the accounting information systems course. Review of Business Information Systems (RBIS), 6(1), 1-6.

6. Romney, M., \& Steinbart, P. (2008). Accounting information systems (11th ed.). Upper Saddle River, NJ: Prentice Hall.

7. Simkin, M. G. (2011). The design, development, and evaluation of database projects in accounting classes. Review of Business Information Systems (RBIS), 9(1), 17-30.

8. Stewart, J. R. (2011). Incorporating a database management project in an AIS course using access 2000. Review of Business Information Systems (RBIS), 4(4), 47-56. 


\section{NOTES}

